\title{
DEGREE REDUCTION OF B-SPLINE CURVES
}

\author{
BYUNG-GOOK LEE AND YUNBEOM PARK
}

\begin{abstract}
An algorithmic approach to degree reduction of B-spline curves is presented. The new algorithms are based on the blossoming process and its matrix representation. The degree reduction of Bspline curves are obtained by the generalized least square method. The computations are carried out by minimizing the $L_{2}$ distance between the two curves.
\end{abstract}

AMS Mathematics Subject Classification : 65D17, 68U05

Key words and phrases : Degree Reduction, B-spline, Blossoming, Least Square Method

\section{Introduction}

Degree elevation and reduction of B-spline curves are well understood and several algorithms are published $[9,10,11,12,13,14]$. From a software engineering point of view, it is desirable to implement a simple and easyto-understand algorithm. This approach was taken by Piegl and Tiller([10, $11,12]$, who implemented the simplest algorithm; they decomposed the Bspline curve into piecewise Bézier curves, reduced the degree of each Bézier piece, and then composed the piecewise Bézier curves into B-spline curves. We describe here the modified form of Piegl and Tiller's degree reduction algorithm. The procedure allow to reduce the degree from $n$ to $m$ in one step. The new algorithms are based on the blossoming analysis $[2,6,15,16]$ and matrix representation of the degree elevation process[8].

Since a B-spline curve is a piecewise polynomial curve, it is possible to elevate its degree from $p$ to $p+r$. That is, there must exist control points

Received, . Revised, .

(c) 200x Korean Society for Computational \& Applied Mathematics and Korean SIGCAM

This work was supported by Dongseo University and Seowon University, grant 2000. 
$\tilde{P}$ and a knot vector $\tilde{U}$ for the degree elevated curve such that

$$
C_{P}^{n}(u)=C_{\tilde{P}}^{\tilde{n}}(u)=\sum_{i=0}^{\tilde{n}} \tilde{P}_{i} N_{i, p+r}(u) .
$$

The curve $C_{P}^{n}(u)$ and $C_{\tilde{P}}^{\tilde{n}}(u)$ are geometrically and parametrically same. The computing of $\tilde{n}, \tilde{P}$ and $\tilde{U}$ is referred to as degree elevation. The knot vector $\tilde{U}$ and the number of points $\tilde{n}$ can easily be computed as follows.

Assume that $U$ has the form

$$
U=\{\underbrace{a, a, \ldots, a}_{p+1}, \underbrace{u_{1}, \ldots, u_{1}}_{m_{1}}, \ldots, \underbrace{u_{s}, \ldots, u_{s}}_{m_{s}}, \underbrace{b, b, \ldots, b}_{p+1}\}
$$

where the end knots $a$ and $b$ are repeated with multiplicity $p+1$, the interior knots $u_{i}$ are repeated with multiplicity $m_{i}$ and $s$ is the number of distinct interior knots. Since the curve $C_{P}^{n}(u)$ is $C^{p-m_{i}}$-continuous at the knot of multiplicity $m_{i}, C_{\tilde{P}}^{\tilde{n}}(u)$ must have the same continuity. Consequently, the new vector must take the form

$$
\tilde{U}=\{\underbrace{a, a, \ldots, a}_{p+r+1}, \underbrace{u_{1}, \ldots, u_{1}}_{m_{1}+r}, \ldots, \underbrace{u_{s}, \ldots, u_{s}}_{m_{s}+r}, \underbrace{b, b \ldots, b}_{p+r+1}\}
$$

which gives $\tilde{n}=n+(s+2) r$.

The computation of $\tilde{P}$ can be done by the procedure as follows[8]:

(1) Decompose the B-spline curve into piecewise Bézier curves by using decomposition matrix $M_{d}$.

(2) Elevate the degree of each Bézier piece by using elevation matrix $M_{e}$.

(3) Make the B-spline curve from the piecewise Bézier segment by using composition matrix $M_{c}$.

Lee and Park[8] presented the algorithms for computing the matrices $M_{d}$, $M_{e}, M_{c}$. The degree elevation process of the B-spline curves is represented as the elevation matrix $M$, where

$$
M=M_{c} M_{e} M_{d}
$$

\section{Degree Reduction}

In general degree reduction of B-spline curves address the following problem. 
Problem 1 (Degree Reduction). Let $\left\{P_{i}\right\}_{i=0}^{n}$ be a given set of control points which define the B-spline curve

$$
C_{P}^{n}(u)=\sum_{i=0}^{n} P_{i} N_{i, p}(u), a \leq u \leq b
$$

of degree $p$. Then find another points set $\left\{Q_{i}\right\}_{i=0}^{l}$ defining the approximating $B$-spline curve $C_{Q}^{l}(u)$ of lower degree $q<p$ so that a suitable distance function $d\left(C_{P}^{n}, C_{Q}^{l}\right)$ between $C_{P}^{n}$ and $C_{Q}^{l}$ is minimized.

The schemes for degree reduction depend on the choice of the distance function and the requirement of the solution to be either best or only nearly best relative to the distance function. For the degree reduction of any given curve, we must compute a distance of two B-spline curves. The most appropriate metric in geometrical terms would be the Hausdorff distance[3, 4]. Suppose $(M, d)$ is a metric space with subsets $A$ and $B$. We define the Hausdorff metric $d_{H}$ by

$$
d_{H}(A, B)=\max \left\{\sup _{x \in A} d(x, B), \sup _{y \in B} d(y, A)\right\},
$$

where

$$
d(x, B)=\inf _{y \in B} d(x, y) .
$$

If we regard a plane curve as simply a locus of points without any underlying parameterizations, the Hausdorff metric for two such curves is essentially the radius of the largest circle with its center on one curve and touching the other curve. For general parametric curves, this measure is truly independent of the relative parameterizations of two curves. Emery[5] presents a method for explicit computation of Hausdorff metric for piecewise linear curves, but the computation of Hausdorff distance $d_{H}$ of two nonlinear curves is not so easy. So we solve the degree reduction problem of B-spline curves with respect to the $L_{2}$ distance.

Replacing the distance function $d$ in the Problem 1 as the $L_{2}$ distance $d_{L S}$, then the Problem 1 can be rewritten as follows:

Problem 2 ( $L_{2}$ Degree Reduction). Find another points set $\left\{Q_{i}\right\}_{i=0}^{l}$ defining the approximating $B$-spline curve $C_{Q}^{l}(u)$ of lower degree $q<p$ so that 
the least squares distance function

$$
d_{L S}\left(C_{P}^{n}, C_{Q}^{l}\right)=\sqrt{\int_{a}^{b}\left\|C_{P}^{n}(u)-C_{Q}^{l}(u)\right\|^{2} d u}
$$

between $C_{P}^{n}$ and $C_{Q}^{l}$ is minimized on the interval $[a, b]$ where $\|\cdot\|$ denotes the Euclidean distance.

Computing of $l, Q$ and $V$ is referred to as degree reduction. The knot vector $V$ and $l$ can easily be computed as follows. Assume that $U$ has the form

$$
U=\{\underbrace{a, a, \ldots, a}_{p+1}, \underbrace{u_{1}, \ldots, u_{1}}_{m_{1}}, \ldots, \underbrace{u_{s}, \ldots, u_{s}}_{m_{s}}, \underbrace{b, b, \ldots, b}_{p+1}\}
$$

Since degree reduction is thought of as the inverse of degree elevation, the new vector becomes of the form

$$
V=\{\underbrace{a, a, \ldots, a}_{p-r+1}, \underbrace{u_{1}, \ldots, u_{1}}_{m_{1}-r}, \ldots, \underbrace{u_{s}, \ldots, u_{s}}_{m_{s}-r}, \underbrace{b, b, \ldots, b}_{p-r+1}\}
$$

which gives $l=n-(s+2) r$, where $r=p-q$. The computation of $Q$ has been done in the past by applying a global approximation method. For developing the method, we compute the $L_{2}$ distance function $d_{L S}$.

We first consider the computation of the $L_{2}$ distance of two Bézier curves. Let two sets $\left\{A_{i}\right\}_{i=0}^{n}$ and $\left\{B_{i}\right\}_{i=0}^{n}$ represent the control polygons of two different Bézier curves of the same degree $n$. Then, the $L_{2}$ distance[7] between two Bézier curves $a^{n}$ and $b^{n}$ is

$$
d_{L S}\left(a^{n}, b^{n}\right)=\sqrt{D^{t} Q_{n} D},
$$

where $D=A-B, A=\left(A_{0}, \ldots, A_{n}\right)^{t}, B=\left(B_{0}, \ldots, B_{n}\right)^{t}$, and $Q_{n}$ be the $(n+1) \times(n+1)$ matrix with elements

$$
q_{i, j}=\frac{1}{2 n+1} \frac{\left(\begin{array}{c}
n \\
i
\end{array}\right)\left(\begin{array}{c}
n \\
j
\end{array}\right)}{\left(\begin{array}{c}
2 n \\
i+j
\end{array}\right)}, \quad\left\{\begin{array}{l}
i=0,1, \ldots, n \\
j=0,1, \ldots, n .
\end{array}\right.
$$

Let us now consider the B-spline curves, If two sets $\left\{A_{i}\right\}_{i=0}^{n}$ and $\left\{B_{i}\right\}_{i=0}^{n}$ represent the control polygons of two different $\mathrm{B}$-spline curves of degree $p$ over a knot vector

$$
U=\{\underbrace{a, a, \ldots, a}_{p+1}, \underbrace{u_{1}, \ldots, u_{1}}_{m_{1}}, \ldots, \underbrace{u_{s}, \ldots, u_{s}}_{m_{s}}, \underbrace{b, b, \ldots, b}_{p+1}\}
$$


denoted by $C_{A}^{n}$ and $C_{B}^{n}$. Since the two B-spline curve are defined over the same knot vector $U$, we can decompose the two curves with same number of curve segments. Moreover, the two Bézier segments are defined over the same segments. Then we have,

$$
d_{L S}\left(C_{A}^{n}, C_{B}^{n}\right)^{2}=D^{t} M_{D}^{t} Q_{p, s} M_{D} D
$$

where $D=A-B$ is $(n+1)$-vector, $M_{D}$ is the $(p(s+1)+1) \times(n+1)$ decomposition matrix [8] and the $Q_{p, s}$ is $(p(s+1)+1) \times(p(s+1)+1)$ square matrix. The $Q_{p, 0}$ is $Q_{p}$ in (1) and $Q_{p, s}(s>0)$ is a block structured matrix with overlapping the one elements, for example,

$$
Q_{p, 1}=\left(\begin{array}{ccccccc}
q_{0,0} & q_{0,1} & \ldots & q_{0, p} & & & \\
q_{1,0} & q_{1,1} & \ldots & q_{1, p} & & & \\
\vdots & \vdots & \ddots & \vdots & & & \\
q_{p, 0} & q_{p, 1} & \ldots & q_{p, p}+q_{0,0} & q_{0,1} & \ldots & q_{0, p} \\
& & & q_{1,0} & q_{1,1} & \ldots & q_{1, p} \\
& & & \vdots & \vdots & \ddots & \vdots \\
& & & q_{p, 0} & q_{p, 1} & \ldots & q_{p, p}
\end{array}\right)
$$

So, the Problem 2 can be rewritten as follows with the distance $d_{L S}$ in (2):

Problem 3 ( $L_{2}$ Degree Reduction). Find the control points set $\left\{Q_{i}\right\}_{i=0}^{l}$ so that the least squares distance function

$$
d_{L S}\left(C_{P}^{n}, C_{Q}^{l}\right)^{2}=d_{L S}\left(C_{P}^{n}, C_{\tilde{Q}}^{\tilde{l}}\right)^{2}=D^{t} M_{D}^{t} Q_{p, s} M_{D} D
$$

between $\left\{P_{i}\right\}_{i=0}^{n}$ and $\left\{\tilde{Q}_{i}\right\}_{i=0}^{\tilde{l}}$ is minimized, where $\tilde{l}=n$.

In order to develope the reduction scheme, we compute the

$$
\begin{aligned}
& D^{t} M_{D}^{t} Q_{p, s} M_{D} D \\
= & {[P-M Q]^{t} M_{D}^{t} Q_{p, s} M_{D}[P-M Q] } \\
= & P^{t} M_{D}^{t} Q_{p, s} M_{D} P-2 Q^{t} M^{t} M_{D}^{t} Q_{p, s} M_{D} P+Q^{t} M^{t} M_{D}^{t} Q_{p, s} M_{D} M Q
\end{aligned}
$$

where $M$ is $\mathrm{B}-$-spline degree elevation matrix in [8].

One method of obtaining the vector $Q$ is so-called method of least squares[1]. This method consists of minimizing $D^{t} M_{D}^{t} Q_{p, s} M_{D} D$ with respect to $Q$. Choosing as the estimator $\hat{Q}$ that value of $Q$ which minimize $D^{t} M_{D}^{t} Q_{p, s} M_{D} D$ involves differentiating $D^{t} M_{D}^{t} Q_{p, s} M_{D} D$ with respect to the elements of $Q$. 
Equating $\partial\left(D^{t} M_{D}^{t} Q_{p, s} M_{D} D\right) / \partial Q$ to zero and writing the resulting equations in terms of $\hat{Q}$, we find that these equations are

$$
M^{t} M_{D}^{t} Q_{p, s} M_{D} M \hat{Q}=M^{t} M_{D}^{t} Q_{p, s} M_{D} P .
$$

They are known as the normal equations.

Lee and Park[8] show that $M_{c}=\left(M_{D}^{t} M_{D}\right)^{-1} M_{D}^{t}$. Thus

$$
M_{D} M=M_{D} M_{c} M_{e} M_{d}=M_{D}\left(M_{D}^{t} M_{D}\right)^{-1} M_{D}^{t} M_{e} M_{d}=M_{e} M_{d},
$$

and we have

$$
M_{d}^{t} M_{e}^{t} Q_{p, s} M_{e} M_{d} \hat{Q}=M_{d}^{t} M_{e}^{t} Q_{p, s} M_{D} P .
$$

Since $M_{e}$ and $M_{d}$ have full column rank, $M_{e} M_{d}$ has full column rank, and from the definition of the matrix $Q_{p, s}$ and mathematical induction, all the upper left submatrices of the matrix $Q_{p, s}$ have positive determinants. Hence, the matrix $Q_{p, s}$ is real symmetric positive definite. Thus, $M_{d}^{t} M_{e}^{t} Q_{p, s} M_{e} M_{d}$ is nonsingular[17], we have the unique solution,

$$
\hat{Q}=\left(M_{d}^{t} M_{e}^{t} Q_{p, s} M_{e} M_{d}\right)^{-1} M_{d}^{t} M_{e}^{t} Q_{p, s} M_{D} P .
$$

An computed example for degree reduction of a B-spline curve from degree 7 to degree 5 is illustrated in Figure 1 . The Figure 2 shows an example of reducing the degree from degree 5 to degree 3 with the original knot vector $U$. The solid control points curve is the given curve and the circle control points curve is the degree reduced curve. In Figure 3, we have computed the degree reduced B-spline curve as treated in FigurE 2 with refining the knot vector $U$ to $U^{\prime}$, where

$$
\begin{aligned}
U^{\prime}=\quad & \{0,0,0,0,0, .25, .25, .25, .5, .5, .5, .75, .75, .75,1,1,1, \\
& 1.25,1.25,1.25,1.5,1.5,1.5,1.75,1.75,1.75,2,2,2,2,2\}
\end{aligned}
$$

The quality of the approximate curve can be improved by introducing extra knots with multiplicity of at least 3 .

\section{REFERENCES}

[1] T.S. Arthanari, Y. Dodge, Mathematical programming in statistics (John Wiley \& Sons, New York, 1981).

[2] P.J. Barry, An Introduction to Blossoming, in: R.N. Goldman, T. Lyche, Ed., Knot Insertion and Deletion Algorithms for B-spline Curves and Surface, (SIAM, 1993), $1-10$. 


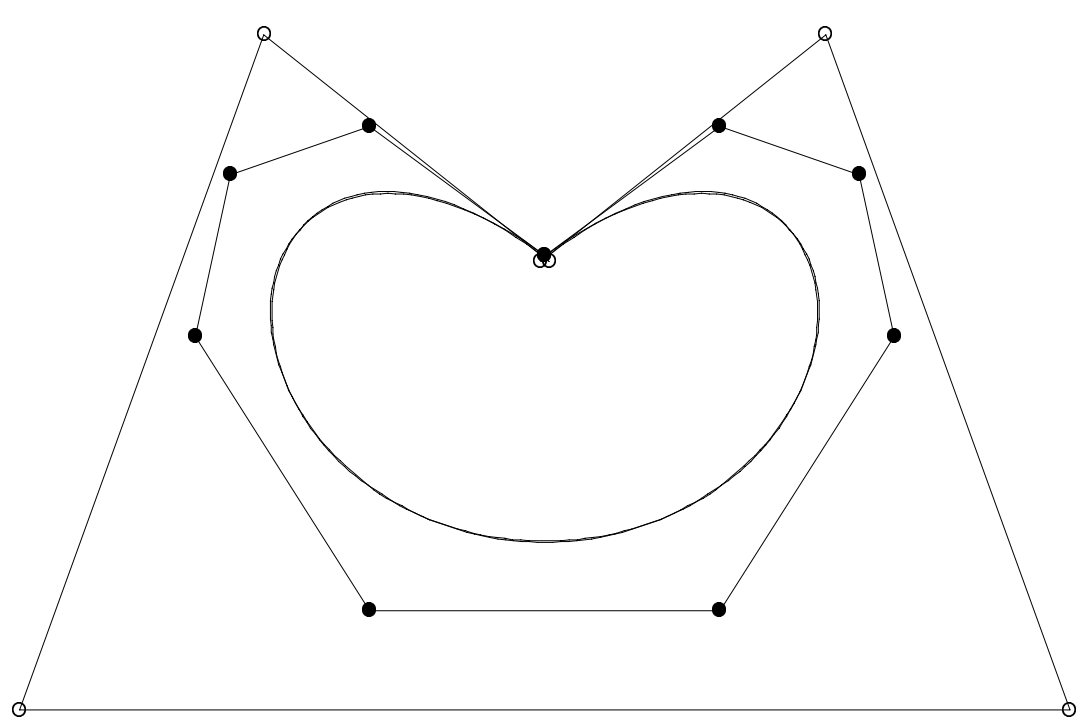

Figure 1. Degree reduction example(from degree 7 to $5)$. The original knot vector is $U=\{0,0,0,0,0,0,0,1,1$, $1,2,2,2,2,2,2,2\}$ and degree reduced control points are circle points.

[3] W.L.F. Degen, Best approximations of parametric curve by spline, in: T. Lyche, L.L. Schumaker, Ed., Mathematical Method in Computer Aided Design II (Academic Press, New York, 1992).

[4] J. Dugundji, Topology, (Allyn and Bacon, Boston, 1966).

[5] J.D. Emery, The definition and computation of a metric on plane curves, Comput. Aided Des. 18(1986), 25-28.

[6] G. Farin, NURBS Curves and Surfaces : from projective geometry to practical use, (AK Peters, Ltd., Wellesley, 1995).

[7] B.G. Lee and Y. Park, The Distance for Bézier Curves and Degree reduction, Bull. Australian Math. Soc. 56(1997), 507-515.

[8] B.G. Lee and Y. Park, The Degree elevation of B-spline curves and its matrix representation, J. KSIAM, 4 (2000), 1-9.

[9] W. Liu, A simple, efficient degree rasing algorithm for B-spline curves, Comput. Aided Geom. Des. 14(1997), 693-698.

[10] L. Piegl, W. Tiller, Software-engineering approach to degree elevation of B-spline curves, Comput. Aided Des. 26(1994), 17-28.

[11] L. Piegl, W. Tiller, Algorithm for degree reduction of B-spline curves, Comput. Aided Des. 27(1995), 101-110. 


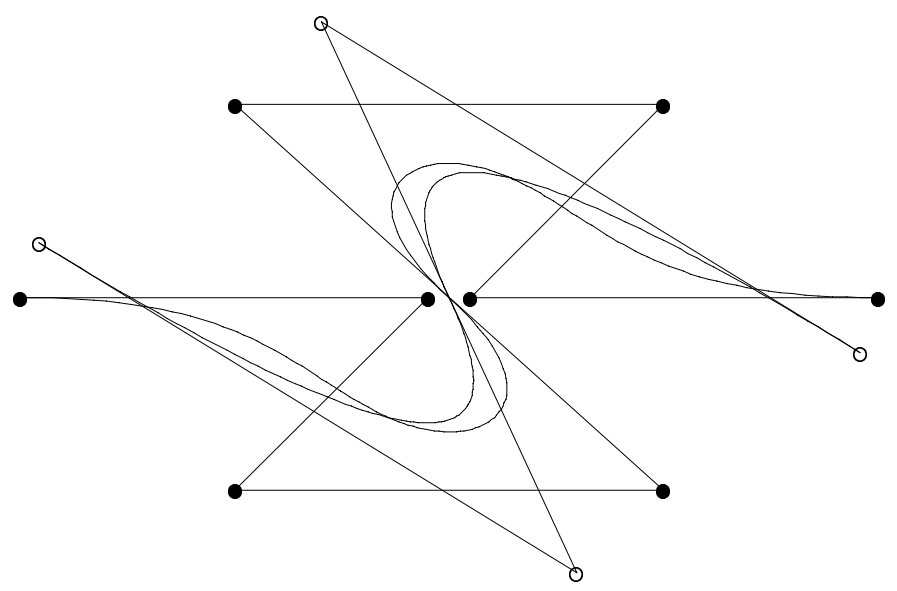

Figure 2. Degree reduction example(from degree 5 to 3 ). The original knot vector is $U=\{0,0,0,0,0,1,1,1$, $2,2,2,2,2\}$.

[12] L. Piegl, W. Tiller, The NURBS Book, (Springer-Verlag, 1995).

[13] H. Prautzsch, Degree elevation of B-spline curves, Comput. Aided Geom. Des. 2(1984), 193-198.

[14] H. Prautzsch, B. Piper, A fast algorithm to raise the degree of B-spline curves, Comput. Aided Geom. Des. 8(1991), 253-266.

[15] L. Ramshaw, Blossoming : a connect-the-dots approach to splines, (Technical report, Digital Systems Research Center, Palo Alto, 1987).

[16] L. Ramshaw, Blossoms are polar forms, Comput. Aided Geom. Des. 6(1989), 323359 .

[17] S.R. Searle, Linear Regression Analysis, (John Wiley \& Sons, New York, 1977).

Byung-Gook Lee is an assistant professor in the Division of Internet, Dongseo University, Pusan, Korea. He received the BS in Mathematics from Yonsei University in 1987. He continued his graduated studies at Korea Advanced Institue of Science and Technology, Korea, where he received the MS and PhD degrees in Applied Mathematics in 1989 and 1993. His research interests are in Computer Aided Geometric Modeling and Computer Graphics.

Division of Internet Engineering, Dongseo University, Pusan 617-716, Korea 


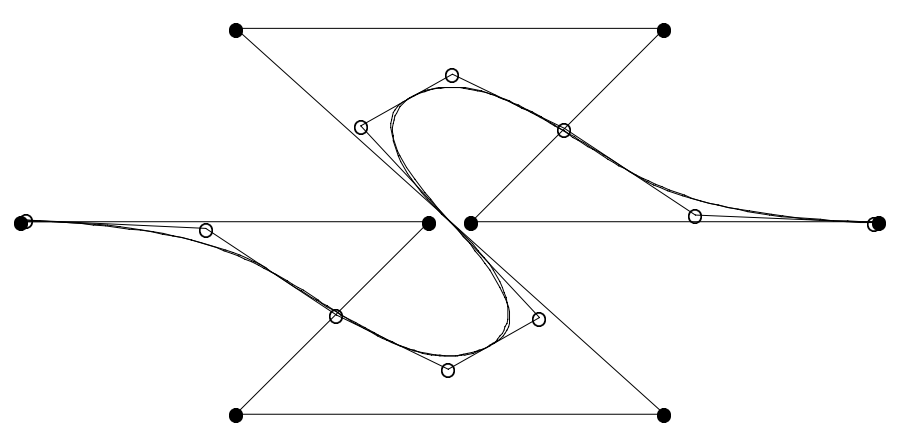

FiguRE 3. Degree reduction example(from degree 5 to 3 ) after refining the knot vector to $U^{\prime}$.

e-mail: lbg@dongseo.ac.kr

Yunbeom Park is an associate professor at the Seowon University, Chongju, Korea. His research interests are in the Computer Aided Geometric Modeling and Numerical approximation theory. He received the BS in Mathematics Education from the Seoul National University in 1986. He continued his graduated studies at Korea Advanced Institue of Science and Technology, Korea, where he received the MS and PhD degrees in Applied Mathematics in 1988 and 1994. He worked at the Sindoricoh company from 1988 to 1991

Department of Mathematics Education, Seowon University, Chongju 361-742, Korea e-mail: ybpark@seowon.ac.kr 